\title{
Corticorelin Ovine Triflutate
}

National Cancer Institute

\section{Source}

National Cancer Institute. Corticorelin Ovine Triflutate. NCI Thesaurus. Code C47463.

A trifluoroacetate salt of ovine corticotropin-releasing hormone (CRH), a 41 amino acids peptide similar to human $\mathrm{CRH}$, used as a diagnostic agent. Corticorelin ovine triflutate is a potent stimulator of adrenocorticotropic hormone (ACTH), secreted from the anterior pituitary gland. In turn, ACTH stimulates cortisol production from the adrenal cortex and is regulated by a negative feedback mechanism. Corticorelin ovine triflutate is used as a diagnostic tool in differentiating pituitary and ectopic production of ACTH. 